\title{
Neutron Lifetime and Dark Decays of the Neutron and Hydrogen
}

\author{
Zurab Berezhiani 1,2 \\ ${ }^{1}$ Dipartimento di Fisica e Chimica, Università di L'Aquila, 67100 Coppito, L'Aquila, Italy \\ ${ }^{2}$ INFN, Laboratori Nazionali del Gran Sasso, 67010 Assergi, L'Aquila, Italy
}

\begin{abstract}
The neutron, besides its $\beta$-decay $n \rightarrow p e \bar{v}_{e}$, might have a new decay channel $n \rightarrow n^{\prime} X$ into mirror neutron $n^{\prime}$, its nearly mass degenerate twin from parallel dark sector, and a massless boson $X$ which can be ordinary and mirror photons or some more exotic particle. Such an invisible decay could alleviate the tension between the neutron lifetimes measured in the beam and trap experiments. I discuss some phenomenological and astrophysical consequences of this scenario, which depends on the mass range of mirror neutron $n^{\prime}$. Namely, the case $m_{n^{\prime}}<m_{p}+m_{e}$ leads to a striking possibility that the hydrogen atom ${ }^{1} \mathrm{H}$ (protium), constituting 75 per cent of the baryon mass in the Universe, could in fact be unstable: it can decay via the electron capture into $n^{\prime}$ and $v_{e}$, with relatively short lifetime $\sim 10^{21} \mathrm{yr}$ or so. If, instead $m_{n^{\prime}}>m_{p}+m_{e}$, then the decay $n^{\prime} \rightarrow p e \bar{v}_{e}$ is allowed and $n^{\prime}$ can represent an unstable dark matter component with rather large lifetime exceeding the age of the Universe. Nevertheless, this decay would produce substantial diffuse gamma background. The dark decay explanation of the lifetime puzzle, however, has a tension with the last experimental results measuring $\beta$-asymmetry in the neutron decay.
\end{abstract}

Keywords:

DOI: 10.31526/LHEP.1.2019.118

1. The neutron, a long-known particle which constitutes half of the mass in our bodies, may still reserve many surprises. While the free neutron is unstable, there still remains a problem to understand what is its true lifetime. According to Standard Model (and common wisdom of baryon conservation) the neutron can have only $\beta$-decay $n \rightarrow p e \bar{v}_{e}$ (including the subdominant daughter branch of radiative decay $n \rightarrow p e \bar{v}_{e} \gamma$ with the photon emission). Hence, its lifetime can be measured in two different ways, known as the trap and beam methods. The trap experiments are based on disappearance of the ultra-cold neutrons (UCN) stored in material or magnetic traps. They measure the true lifetime $\tau_{n}$, equivalent to its total decay width $\Gamma_{n}=\tau_{n}^{-1}$, via counting the survived UCN for different storage times and reproducing the exponential time dependence $\exp \left(-t / \tau_{n}\right)$ after accurately estimating and subtracting other effects of the UCN losses related to the wall absorptions, upscattering etc. The beam experiments are the appearance experiments, measuring the $\beta$-decay width $\Gamma_{\beta}$ by counting the protons produced via decay $n \rightarrow p e \bar{v}_{e}$ in the monitored beam of cold neutrons. Clearly, in the absence of new physics both methods should measure the same value, $\Gamma_{n}=\Gamma_{\beta}$.

However, as it was noticed quite a time ago [1], the neutron lifetimes measured with two methods have some discrepancy. Careful re-analysis of the previous experimental results and new measurements with increased precision rendered this discrepancy more evident [2].

Fig. 1 summarizes results of the neutron lifetime measurements performed from 1988 till now (experiments which results were removed and the ones reporting error-bars exceeding $10 \mathrm{~s}$ are not included). The trap experiments, Refs. $[3,4,5,6,7,8,9,10,11,12,13]$, are in good agreement with each other, and their average reads

$\tau_{\text {trap }}=(879.4 \pm 0.5) \mathrm{s}$.

The results of beam experiments, Refs. $[14,15,16]$, also are in fine agreement and their average yields

$\tau_{\text {beam }}=(888.1 \pm 2.0) \mathrm{s}$.

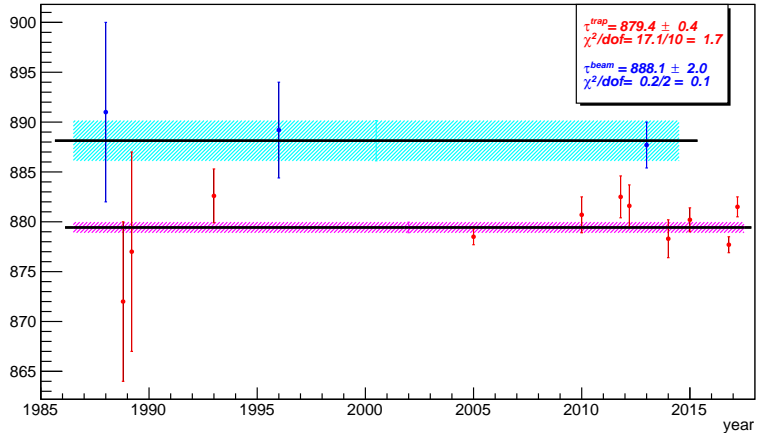

FIGURE 1: Results of the trap (red) and beam (blue) measurements and respective averages.

The discrepancy is about $9 \mathrm{~s}$. Formally the beam result (2) is $4 \sigma$ away from the trap result (1).

It is instructive to follow the time evolution of the neutron lifetime as it is reflected in the Particle Data Group (PDG) editions of last years. PDG 2010 [17] summarises available experimental results and adopts the world average $\tau=885.7 \pm 0.8 \mathrm{~s}$. However, it discards the result of most accurate measurements reported by the Serebrov's group in 2005, $\tau=878.5 \pm 0.8 \mathrm{~s}$ [6], with the following comment: "SEREBROV 05 result is $6.5 \sigma$ deviations from our average of previous results and $5.6 \sigma$ deviations from the previous most precise result (that of ARZUMANOV 00)", since by that time results of all beam and trap experiments (excluding that of Serebrov's) were in good agreement. However, already the next edition, PDG 2012 [18], adopted a world average $\tau=880.1 \pm 1.1 \mathrm{~s}$, rather distant from the previous PDG 2010 one, and also with larger error-bars. This value suffered only minor changes in following PDG editions [19, 20]. Namely, PDG 2018 quotes its value as $\tau=880.2 \pm 1.0 \mathrm{~s}$ [21], without the latest results of Refs. [10,12,13] being included. What has happened between PDG 2010 and PDG 2012 editions? First, the result of Serebrov's experiment [6] was included, and second, in 2010 Serebrov and Fomin critically reanalyzed the results of all trap experiments performed before 2005 and found a system- 
atic error of about $6 \mathrm{~s}$ [22]. In consequence, many experimental groups themselves reevaluated their previous results and adopted new corrected values (see Refs. [8, 9]). By the time, results of new trap measurements [7] were also published, all consistent with the previously discarded result of Ref. [6].

On the other side, the beam results showed quite an opposite trend. The re-analysis of previous beam measurements brought to larger value of $\tau_{\beta}$ with smaller error-bars [16]. In this way, the discrepancy between the neutron lifetimes measured in the trap and beam experiments became rather evident what renders the situation more enigmatic.

2. The fact that $\tau_{\text {beam }}(2)$ is larger than $\tau_{\text {trap }}(1)$ with about one percent difference:

$\Delta \tau_{n}=\tau_{\text {beam }}-\tau_{\text {trap }}=(8.7 \pm 2.1) \mathrm{s}$,

may suggest that apart of usual $\beta$-decay $n \rightarrow p e \bar{v}_{e}$, the neutron may have a new decay channel, invisible or semi-invisible (i.e., in principle detectable but not yet excluded experimentally). In this case, the trap experiments would measure the neutron total decay width, $\Gamma_{n}=\Gamma_{\beta}+\Gamma_{\text {new }}=\tau_{\text {trap }}^{-1}=7.485 \times 10^{-28} \mathrm{GeV}$, where $\beta$-decay width $\Gamma_{\beta}=\tau_{\text {beam }}^{-1}$ measured by beam experimentts should constitute a dominant part of it, with the branching ratio $\operatorname{Br}\left(n \rightarrow p e \bar{v}_{e}\right)=\Gamma_{\beta} / \Gamma_{n}=\tau_{\text {trap }} / \tau_{\text {beam }}=0.99$. Therefore, the new decay channel with about 1 per cent branching ratio would suffice for resolving the discrepancy. Namely, given the error-bars in (1) and (2), for reconciling the situation at about $1 \sigma$ level one would need

$\Gamma_{\text {new }}=(7 \pm 2) \times 10^{-30} \mathrm{GeV}$.

For example, the neutron could decay in two invisible particles, $n \rightarrow n^{\prime}+X$, where $n^{\prime}$ is a "dark" fermion with a mass $m_{n}^{\prime}<$ $m_{n}$ and $X$ is a massless (or light enough) "dark" boson, while the "yet-invisible" mode could be $n \rightarrow n^{\prime}+\gamma$ with the photon emission.

Clearly, new particle $n^{\prime}$ cannot be arbitrarily light, and the mass splitting $\Delta m=m_{n}-m_{n^{\prime}}$ is limited by the stability of chemical elements with precision of about a MeV. While allowing the decay $n \rightarrow n^{\prime}+X$ for a free neutron, i.e., $m_{n^{\prime}}<m_{n}$, this decay should be forbidden for a neutron bound in that nuclei which are known to be stable. The strongest bound comes from the stability of ${ }^{9} \mathrm{Be}$ which has a rather fuzzy nuclei, having the minimal neutron separation energy among all stable elements. Transition $n \rightarrow n^{\prime}$, if allowed by phase space, would transform ${ }^{9} \mathrm{Be}, M\left({ }^{9} \mathrm{Be}\right)=8394.79535 \mathrm{MeV}$, into ${ }^{8} \mathrm{Be}$, $\mathrm{M}\left({ }^{8} \mathrm{Be}\right)=7456.89447 \mathrm{MeV}$, which is $\alpha$-unstable with decay time $\sim 10^{-16} \mathrm{~s}$. In fact, the stability of ${ }^{9} \mathrm{Be}$ atom against the removal of extra neutron $n \rightarrow n^{\prime}$ implies that $m_{n^{\prime}}$ should be larger than the mass of ${ }^{9} \mathrm{Be}$ minus twice the mass of ${ }^{4} \mathrm{He}$, $M\left({ }^{4} \mathrm{He}\right)=3728.40132 \mathrm{MeV}$. In this way, one can set a lower limit

$m_{n^{\prime}}>937.992 \mathrm{MeV}$, i.e., $\Delta m_{\max }=1.573 \mathrm{MeV}$

Other stable elements do not give competitive limits, e.g., Deutrium ${ }^{2} \mathrm{H}$ stability implies $m_{n^{\prime}}>M\left({ }^{2} \mathrm{H}\right)-M\left({ }^{1} \mathrm{H}\right)=$ 937.3358 MeV or $\Delta m<2.230 \mathrm{MeV}$, while the limits from other elements are yet weaker.

A sterile particle $n^{\prime}$ so closely degenerate in mass with the neutron, with precision of $\Delta m / m_{n} \sim 10^{-3}$ can be introduced $a d$ $h o c$ as an elementary fermion but this sort of fine tuning does not look very appealing. In addition, for $n^{\prime}$ being an elementary fermion with negligible self-interaction, rapid $n \rightarrow n^{\prime}+X$ transition would have disastrous consequences for the neutron star stability: the stars made of degenerate gas of free fermions can have a maximal mass $M_{\max }=0.627 M_{\odot}\left(1 \mathrm{GeV} / m_{n^{\prime}}\right)^{2}$ [23] which for $m_{n}^{\prime} \simeq m_{n}$ gives $M_{\max } \approx 0.71 M_{\odot} \cdot{ }^{1}$ But the typical masses of neutron stars are about twice as larger, and moreover at least two pulsars of $2 M_{\odot}$ were observed. For achieving that large masses, one has to introduce strong repulsive force between fermions $n^{\prime}$ mediated by a vector boson $I$ with large coupling constant $g$. In this case, the maximal mass can be increased to $M_{\max }=\left(0.7+0.3 y_{n^{\prime}}\right)\left(m_{n} / m_{n^{\prime}}\right)^{2} M_{\odot}$ where the parameter $y=(g / \sqrt{2}) m_{n^{\prime}} / m_{I}$ describes the interaction strength [23]. In the case for the neutrons, this repulsive force can be induced by $\rho-\omega$ mesons, and taking $m_{\rho} \simeq 0.8 \mathrm{GeV}$ and $g_{\rho n} \simeq 13$, one has $y_{n} \simeq 10$ and the compact objects can be as heavy as $3.7 \mathrm{M}_{\odot} .^{2}$ This suggests that for avoiding the neutron star destabilization due to rapid $n \rightarrow n^{\prime} X$ decay, $n^{\prime}$ can be considered as composite fermion subject to short-distance repulsive forces of nearly the same strength as the repulsive forces acting between the neutrons. Namely, one can envisage that $n^{\prime}$ is composed of three hypothetical 'quarks' bounded by some new color $S U$ (3) forces. However, there remains a question why it is so nearly degenerate in mass with the neutron.

In this view, it would be natural to consider new fermion $n^{\prime}$ as a dark twin of the neutron $n$ from a parallel hidden sector, coined as mirror world, which is an identical copy of ordinary particle sector (for reviews, see e.g. Refs. [26, 27]). The identical form of two sectors can be ensured by a discrete symmetry, $Z_{2}$ parity. In this picture, all ordinary particles: the electron $e$, proton $p$, neutrinos $v$ etc., should have exactly mass-degenerate invisible twins: $e^{\prime}, p^{\prime}, v^{\prime}$, etc. which are sterile to our strong and electroweak interactions $S U(3) \times S U(2) \times U(1)$ but have their own gauge interactions $S U(3)^{\prime} \times S U(2)^{\prime} \times U(1)^{\prime}$. Mirror matter, with its features of being baryon-asymmetric, atomic and thus dissipative, can represent part or even entire amount of dark matter in the Universe, with specific implications for the cosmological evolution, formation and structure of galaxies and stars, etc. $[28,29,30]$.

Interestingly, the baryon asymmetries in both ordinary and mirror worlds can be generated by particle processes that violate $B-L$ and $C P$ in both sectors [31] which can explain the relation between the dark and visible matter fractions in the Universe, $\Omega_{B}^{\prime} / \Omega_{B} \simeq 5$. On the other hand, the same interactions can induce mixing phenomena between ordinary and mirror particles. In fact, any neutral particle, elementary or composite, may have a mixing with its mirror twin. E.g., three ordinary neutrinos $v_{e, \mu, \tau}$ can be mixed with their mirror partners $v_{e, \mu, \tau}^{\prime}$ which in fact are most natural candidates for the role of sterile neutrinos [32].

The mixing between the neutron $n$ and its mirror twin $n^{\prime}$ was introduced in Ref. [33], assuming that two states are ex-

\footnotetext{
${ }^{1}$ In fact, this is well-known since 1939 from the original work of Oppenheimer and Volkoff [24] which obtained $M_{\max } \simeq 0.7 M_{\odot}$ for maximal mass neutron stars considering neutrons as degenerate Fermi gas (see also in textbook of Shapiro and Teukolsky [25]).

${ }^{2}$ Of course, for real neutron stars this is only a rough approximation. In a realistic approach, along with the repulsive vector interaction also attractive scalar interaction should be included which reduces $M_{\max }$. Unfortunately, the true equation of state of dense nuclear matter is of difficult determination and real value of maximal mass of neutron stars remains unknown, though experimentally we know that it should be larger than $2 M_{\odot}$.
} 
actly degenerate in mass, and its astrophysical and cosmological implications were discussed in Refs. [33, 34]. This mixing is similar, and perhaps complementary, to neutron-antineutron $(n-\bar{n})$ mixing [35]. However, in difference from the latter, it is not restricted by the nuclear stability limits since $n-n^{\prime}$ transition for a neutron bound in nuclei cannot take place simply because of energy conservation [33]. Possible experimental strategies for searching $n-n^{\prime}$ oscillation were discussed in Refs. [31,36], and the results of several dedicated experiments can be found in Refs. [37].

In this paper, we consider the situation when mirror symmetry is softly or spontaneously broken and $n$ and $n^{\prime}$ states are not exactly degenerate in mass but have a mass splitting of about a MeV, so that $n \rightarrow n^{\prime} X$ decay of free neutron becomes possible. This concept suggests intriguing connection between the neutron lifetime and dark matter puzzles. In particular, dark matter can present entirely in the form of mirror neutrons, without an atomic component, if mirror proton $p^{\prime}$ is heavier than mirror neutron $n^{\prime}$, and it decays as $p^{\prime} \rightarrow n^{\prime} \bar{e}^{\prime} v_{e}^{\prime}$. We shall discuss implications of $n \rightarrow n^{\prime}$ decay in the light of the neutron lifetime puzzle and other issues as are the matter stability, dark matter decay, etc.

3. One can consider a theory based on the product $G \times G^{\prime}$ of two identical gauge factors (Standard Model $S U(3) \times S U(2) \times$ $U(1)$ or some of its extension), ordinary $(\mathrm{O})$ particles belonging to $G$ and mirror (M) particles to $G^{\prime}$ (mirror Standard Model $S U(3)^{\prime} \times S U(2)^{\prime} \times U(1)^{\prime}$ or its equivalent extension).

In the Standard Model, the quark fields are represented as Weyl spinors, the left-handed (LH) ones transforming as weak isodoublets and the right-handed $(\mathrm{RH})$ ones as isosinglets, whereas the anti-quark fields $\bar{q}$ which are $\mathrm{CP}$ conjugated to $q\left(\bar{q}_{R, L}=C \gamma_{0} q_{L, R}^{*}\right)$ have the opposite chiralities and opposite gauge charges:

$$
\begin{gathered}
q_{L}=\left(\begin{array}{l}
u_{L} \\
d_{L}
\end{array}\right), \quad q_{R}=u_{R}, d_{R}, \\
\bar{q}_{R}=\left(\begin{array}{c}
\bar{u}_{R} \\
\bar{d}_{R}
\end{array}\right), \quad \bar{q}_{L}=\bar{u}_{L}, \bar{d}_{L}
\end{gathered}
$$

(the family indices are suppressed, and the lepton fields are omitted for brevity). In addition, we assign to quarks $q_{L}, u_{R}, d_{R}$ a global baryon charge $\mathrm{B}=1 / 3$. Then antiquarks $\bar{q}_{R}, \bar{u}_{L}, \bar{d}_{L}$ have $\mathrm{B}=-1 / 3$.

The parallel $\mathrm{M}$ sector $G^{\prime}=S U(3)^{\prime} \times S U(2)^{\prime} \times U(1)^{\prime}$ has the analogous quark content

$$
\begin{aligned}
& q_{L}^{\prime}=\left(\begin{array}{c}
u_{L}^{\prime} \\
d_{L}^{\prime}
\end{array}\right), \quad q_{R}^{\prime}=u_{R}^{\prime}, d_{R}^{\prime} \\
& \bar{q}_{R}^{\prime}=\left(\begin{array}{c}
\bar{u}_{R}^{\prime} \\
\bar{d}_{R}^{\prime}
\end{array}\right), \quad \bar{q}_{L}^{\prime}=\bar{u}_{L}^{\prime}, \bar{d}_{L}^{\prime}
\end{aligned}
$$

For definiteness, we name $\bar{q}_{R}^{\prime}, \bar{u}_{L}^{\prime}, \bar{d}_{L}^{\prime}$ as mirror quarks and assign them a mirror baryon number $\mathrm{B}^{\prime}=1 / 3$. Then mirror antiquarks $q_{L}^{\prime}, u_{R}^{\prime}, d_{R}^{\prime}$ have $\mathrm{B}^{\prime}=-1 / 3$.

The Lagrangian of two systems has a generic form

$\mathcal{L}_{\text {tot }}=\mathcal{L}+\mathcal{L}^{\prime}+\mathcal{L}_{\text {mix }}$

where $\mathcal{L}$ and $\mathcal{L}^{\prime}$ respectively are the Standard Lagrangians of $\mathrm{O}$ and $\mathrm{M}$ sectors, including the gauge, Yukawa and Higgs parts, while $\mathcal{L}_{\text {mix }}$ stands for possible interactions between the particles of two sectors. The identical forms of $\mathcal{L}$ and $\mathcal{L}^{\prime}$ can be ensured by discrete $Z_{2}$ symmetry under the exchange $G \leftrightarrow G^{\prime}$ when all $\mathrm{O}$ particles (fermions, Higgs and gauge fields) exchange places with their $M$ twins ('primed' fermions, Higgs and gauge fields). Such a discrete symmetry can be imposed with or without chirality change between the $\mathrm{O}$ and $\mathrm{M}$ fermions [26]. However, this difference will have no relevance for our further discussion; what is important that this symmetry ensures that the gauge and Yukawa coupling constants are the same in two sectors. Hence, if $Z_{2}$ symmetry between two sectors is unbroken, i.e., $\mathrm{O}$ and $\mathrm{M}$ Higgses $\phi$ and $\phi^{\prime}$ have exactly the same vacuum expectation values (VEVs), then mirror world will be an exact replica of ordinary particle sector, and all $\mathrm{O}$ particles: the electron $e$, proton $p$, neutron $n$ etc., would be exactly mass-degenerate with their $\mathrm{M}$ twins: $e^{\prime}, p^{\prime}, n^{\prime}$, etc.

However, one can envisage a situation when $Z_{2}$ is spontaneously broken. E.g., one can introduce a real scalar field $\eta$ which is odd under $Z_{2}$ symmetry, i.e., transforms as $\eta \rightarrow-\eta$ [28]. If this scalar acquires a non-zero VEV, then its coupling to $\mathrm{O}$ and $\mathrm{M}$ Higgses will give different contributions to their mass terms. In this way, the $\mathrm{O}$ and $\mathrm{M}$ Higgses can get different VEVs, and so the masses of $\mathrm{O}$ and $\mathrm{M}$ quarks would be different.

Let us consider a situation when each of the $O$ and $M$ sectors are represented by the models with two Higgs doublets $\phi_{1,2}$ and $\phi_{1,2}^{\prime}$, responsible for the masses of up and down quarks, as motivated by e.g., supersymmetry. In this case, the couplings of $Z_{2}$-odd scalar $\lambda_{1} \eta\left(\phi_{1}^{\dagger} \phi_{1}-\phi_{1}^{\prime \dagger} \phi_{1}^{\prime}\right)$ and $\lambda_{2} \eta\left(\phi_{2}^{\dagger} \phi_{2}-\right.$ $\left.\phi_{2}^{\prime \dagger} \phi_{2}^{\prime}\right)$, with $\lambda_{1,2}$ being dimensional couplings, would lead to different VEVs, $v_{1}^{\prime} \neq v_{1}$ and $v_{2}^{\prime} \neq v_{2}$. For evading the strong hierarchy problem and related fine tunings, we can assume that all of these four values are in the range of few hundred $\mathrm{GeV}$, with $v_{\text {ew }}=\left(v_{1}^{2}+v_{2}^{2}\right)^{1 / 2}$ determining the (known) ordinary weak scale and $v_{\mathrm{ew}}^{\prime}=\left(v_{1}^{\prime 2}+v_{2}^{\prime 2}\right)^{1 / 2} \neq v_{\mathrm{ew}}$ determining the mirror weak scale; one can take e.g., $\lambda_{1,2} \sim 1 \mathrm{GeV}$ and $\langle\eta\rangle$ in the range of few $\mathrm{TeV}^{3}$ Due to renormalisation group effects, the difference between the $\mathrm{O}$ and $\mathrm{M}$ Higgs VEVs can induce some difference between the QCD scales in two sectors but for $v_{\mathrm{ew}}^{\prime} \sim v_{\mathrm{ew}}$, we expect that $\Lambda_{\mathrm{QCD}}^{\prime} \simeq \Lambda_{\mathrm{QCD}}$. In this case, the light quark masses of both sectors are expected to be of few $\mathrm{MeV}$ and so the mass splitting between $\mathrm{M}$ and $\mathrm{O}$ nucleons can be in the $\mathrm{MeV}$ range.

In particular, one can envisage a situation when $m_{u^{\prime}}>m_{u}$ but $m_{d^{\prime}}<m_{d}$ and $m_{e^{\prime}}<m_{e}$. Let us take a simple example when $v_{1}^{\prime} \simeq 2 v_{1}$ but $v_{2}^{\prime} \simeq v_{2} / 2$. Given that the Yukawa coupling constants in two sectors are the same, for the reference masses or our light quarks $m_{u} \simeq 2 \mathrm{MeV}$ and $m_{d} \simeq 4 \mathrm{MeV}$, the mirror light quarks masses are just inverted, $m_{u}^{\prime} \simeq 4 \mathrm{MeV}$ and $m_{d}^{\prime} \simeq 2$ $\mathrm{MeV}$, while for the electrons we have $m_{e}^{\prime} \simeq m_{e} / 2$. Therefore, it could occur pretty naturally that the mirror neutron and proton have masses different from their ordinary twins by a $\mathrm{MeV}$ or so, but to different sides arranged as $m_{p}^{\prime}>m_{n}>m_{n}^{\prime}>m_{p}$. Namely, if $m_{p^{\prime}}>m_{n^{\prime}}+m_{e^{\prime}}$, then free M proton $p^{\prime}$ would be unstable so that mirror world would contain no hydrogen. However mirror neutron $n^{\prime}$ would be stable and thus represent a self-scattering dark matter with just a perfect cross-section over mass ratio, $\sigma / m_{n} \sim 1 \mathrm{bn} / \mathrm{GeV}$. In the following, we consider this case as a reference model. In other possible situation when $\left|m_{p^{\prime}}-m_{n^{\prime}}\right|<m_{e^{\prime}}$, both $p^{\prime}$ and $n^{\prime}$ will be stable and one would

\footnotetext{
${ }^{3}$ More generically, if two sectors contain scalar fields other than the Higgs doublets $\phi_{1,2}$ and $\phi_{1,2}^{\prime}$, as e.g. color scalars $S$ and $S^{\prime}$ discussed in next section, then the couplings of $Z_{2}$ odd scalar $\lambda \eta\left(S^{\dagger} S-S^{\prime \dagger} S^{\prime}\right)$ would induce different masses for them, $M_{S}^{\prime} \neq M_{S}$. In particular, for achieving e.g. $M_{S} \sim 1 \mathrm{TeV}$ and $M_{S^{\prime}} \sim 100 \mathrm{GeV}$, we would need rather large values of $\lambda$ approaching $\mathrm{TeV}$ scale.
} 
have dark matter in two forms, self scattering component $n^{\prime}$ and dissipative components in the form of mirror hydrogen $\mathrm{H}^{\prime}$ and helium $\mathrm{He}^{\prime}$.

4. Let us concentrate on the system of two neutrons, ordinary $n$ and mirror $n^{\prime}$. The relevant terms of the generic Lagrangian (8) are the low energy effective terms related to their masses and magnetic moments:

$$
\begin{aligned}
& \mathcal{L}=m_{n} \bar{n} n+\frac{\mu_{n}}{2} F_{\mu v} \bar{n} \sigma^{\mu v} n \\
& \mathcal{L}^{\prime}=m_{n^{\prime}} \overline{n^{\prime}} n^{\prime}+\frac{\mu_{n^{\prime}}}{2} F_{\mu v}^{\prime} \overline{n^{\prime}} \sigma^{\mu v} n^{\prime} \\
& \mathcal{L}_{\text {mix }}=\epsilon \bar{n} n^{\prime}+\frac{\kappa_{n n^{\prime}}}{2} F_{\mu v} \bar{n} \sigma^{\mu v} n^{\prime}+\frac{\kappa_{n n^{\prime}}^{\prime}}{2} F_{\mu v}^{\prime} \bar{n} \sigma^{\mu v} n^{\prime}+\text { h.c. }
\end{aligned}
$$

where $F_{\mu \nu}=\partial_{\mu} A_{\nu}-\partial_{\nu} A_{\mu}$ is the electromagnetic field strength tensor, and $F_{\mu \nu}^{\prime}=\partial_{\mu} A_{v}^{\prime}-\partial_{\nu} A_{\mu}^{\prime}$ is the same for mirror electromagnetic field, $m_{n}=939.5654 \mathrm{MeV}$ and $\mu_{n}=-1.912 \mu_{N}$ respectively are the neutron mass and magnetic moment, $\mu_{N}=$ $e / 2 m_{p}$ being the nuclear magneton, and $m_{n^{\prime}}$ and $\mu_{n^{\prime}}$ are those of mirror neutron. We assume that due to $Z_{2}$ breaking, there is a small mass splitting between $n$ and $n^{\prime}$ states, $\Delta m=m_{n}-m_{n^{\prime}} \simeq$ $1 \mathrm{MeV}$. The magnetic moments $\mu_{n}$ and $\mu_{n^{\prime}}$ should also have some tiny difference but this is irrelevant for our discussion and one can safely take $\mu_{n^{\prime}}=\mu_{n}$ as a good approximation. In addition, unlike the case of neutron-antineutron system, transitional magnetic (or electric dipole) moments $\kappa_{n n^{\prime}}$ and $\kappa_{n n^{\prime}}^{\prime}$ between the neutron and mirror neutron are not forbidden by fundamental symmetry reasons [38]. Lagrangians $\mathcal{L}$ and $\mathcal{L}^{\prime}$ in (9) conserve baryon numbers $\mathrm{B}$ and $\mathrm{B}^{\prime}$ separately, while the mass term in $\mathcal{L}_{\text {mix }}$ mixing the states $n(B=1)$ and $n^{\prime}\left(B^{\prime}=1\right)$ conserves the combination of baryon charges $\overline{\mathrm{B}}=\mathrm{B}+\mathrm{B}^{\prime}$.

The mixing term $\mathcal{L}_{\text {mix }}$ can be induced by the effective sixquark operators with different Lorentz structures involving $\mathrm{LH}$ and $R H$ quarks $u_{L, R}, d_{L, R}$ and mirror antiquarks $u_{L, R}^{\prime}, d_{L, R}^{\prime}$ in gauge singlet combinations [33]:

$\frac{1}{\mathcal{M}^{5}}(\bar{u} \bar{d} \bar{d})\left(u^{\prime} d^{\prime} d^{\prime}\right)+$ h.c.

The Lorentz, gauge and family indices are suppressed. These operators transform the neutron state $n$ (three valent quarks udd, $\overline{\mathrm{B}}=1$ ) into mirror neutron $n^{\prime}$ (three mirror quarks $\bar{u}^{\prime} \bar{d}^{\prime} \bar{d}^{\prime}$, again $\overline{\mathrm{B}}=1$ ). Taking the matrix elements $\langle n|u d d| 0\rangle=$ $K \Lambda_{\mathrm{QCD}}^{3} \simeq K \times 0.015 \mathrm{GeV}^{3}$, with $K$ being an order 1 coefficient, and equivalently for the mirror neutron, we obtain the $n-n^{\prime}$ mixing mass as $\epsilon \simeq(K / 2)^{2}\left(10^{10} \mathrm{GeV}^{5} / \mathcal{M}^{5}\right) \times 10^{-10} \mathrm{MeV}$.

Operators (10) can be induced via seesaw-like mechanism $[33,39]$ from the following Lagrangian terms: ${ }^{4}$

$$
\begin{aligned}
& \mathcal{L}=g S u_{R, L} d_{R, L}+h_{a} S^{\dagger} d_{R} N_{R a}+\text { h.c. } \\
& \mathcal{L}^{\prime}=g S^{\prime} u_{R, L}^{\prime} d_{R, L}^{\prime}+h_{a} S^{\prime \dagger} d_{R}^{\prime} N_{R a}^{\prime}+\text { h.c. } \\
& \mathcal{L}_{\text {mix }}=M_{D}^{(a)} N_{R a} N_{R a}^{\prime}+\text { h.c.; } \quad a=1,2, \ldots
\end{aligned}
$$

involving a color-triplet scalar $S(\overline{\mathrm{B}}=-2 / 3)$ with mass $M_{S}$ and and its mirror partner $S^{\prime}(\overline{\mathrm{B}}=2 / 3)$ with mass $M_{S^{\prime}}$. It also involves gauge singlet $\mathrm{RH}$ fermions $N_{R a}$ with $\overline{\mathrm{B}}=-1$ and $N_{R a}^{\prime}$

${ }^{4}$ Color indices and charge conjugation matrix $C$ are suppressed. $u_{L} d_{L}$ can enter in weak isosinglet combination $\epsilon^{\alpha \beta} q_{L \alpha} q_{L \beta}$ where $\alpha, \beta=1,2$ are the weak $S U(2)$ indices. For simplicity we take the constants of couplings $S u_{R} d_{R}$ and $S u_{L} d_{L}$ equal, $g_{L}=g_{R}=g$, and take $K \simeq 2$.

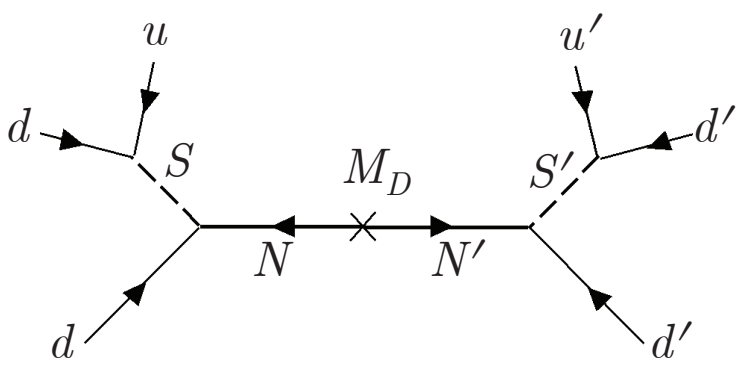

FIGURE 2: Seesaw diagram generating $n-n^{\prime}$ mixing

with $\overline{\mathrm{B}}=1$, so that the mass terms in (11) conserves the combined charge $\overline{\mathrm{B}}=\mathrm{B}+\mathrm{B}^{\prime}$. In fact, these mass terms $M_{D}$ are the Dirac mass terms: one can say that RH components $N_{R}$ of Dirac spinors $N_{L}+N_{R}$ belong to ordinary sector and LH components $N_{L}=C{\overline{N_{R}^{\prime}}}^{T}$ belong to mirror sector.

Integrating out the heavy fermions and scalars, the diagrams shown on Fig. 2 effectively induce operator (10) and for $n-n^{\prime}$ mixing mass, we get

$\epsilon \simeq \frac{g^{2} h^{2} N_{\mathrm{eff}} \times 10^{10} \mathrm{GeV}^{5}}{M_{S}^{2} M_{S^{\prime}}^{2} M_{D}} \times 10^{-10} \mathrm{MeV}$

where $N_{\text {eft }}$ is the effective number of $N, N^{\prime}$ states which takes into account that the latter can have different masses, i.e., $h^{2} N_{\text {eft }} / M_{D}=\sum_{a} h_{a}^{2} / M_{D}^{(a)}$. The masses of $S$ and $S^{\prime}$ are split due to the couplings with $Z_{2}$ odd scalar $\eta, \eta\left(S^{\dagger} S-S^{\prime \dagger} S^{\prime}\right)$, so that $M_{S} \neq M_{S^{\prime}}$. For having large enough $\epsilon$, one has to take into account the LHC limits on the color triplet $S$ involved in the game (for more details, see Ref. [39]). Namely, the first term in $\mathcal{L}(11)$ induces the contact operators $\bar{q} q \bar{q} q$ which are restricted by the compositeness limits. Namely, the LHC limit $\Lambda_{L L, R R}^{-}>22 \mathrm{TeV}$ [21] translates to $\left(M_{S} / g\right)^{2}>0.75 \times 10^{8} \mathrm{GeV}^{2}$ or so which can be saturated for $M_{S} \simeq 1.7 \mathrm{TeV}$ and $g \simeq 0.2$. Therefore, $\epsilon \sim 10^{-10} \mathrm{MeV}$ can be achieved by taking $M_{S^{\prime}} \simeq 50$ $\mathrm{GeV}, M_{D} \simeq 5 \mathrm{GeV}$ and $h^{2} N_{\text {eff }} \sim 10^{2}$. While this parameter space looks rather marginal, it is not excluded by the present experimental bounds.

This mass term induces small mixing between $n-n^{\prime}$, with a mixing angle $\theta=\epsilon / \Delta m$. For our benchmark values $\epsilon=$ $10^{-10} \mathrm{MeV}$ and $\Delta m=1 \mathrm{MeV}$, we have $\theta=10^{-10}$. This mixing in turn induces transitional magnetic moment $\mu_{n n^{\prime}}=\theta \mu_{n}$ between the mass eigenstates $n_{1}=n+\theta n^{\prime}$ and $n_{2}=n^{\prime}-\theta n$, Therefore, the heavier eigenstate $n_{1} \approx n$ can decay into the lighter one $n_{2} \approx n^{\prime}$ with the photon emission:

$\Gamma\left(n \rightarrow n^{\prime} \gamma\right)=\frac{\left|\mu_{n n^{\prime}}\right|^{2}\left(m_{n}^{2}-m_{n^{\prime}}^{2}\right)^{3}}{8 \pi m_{n}^{3}}=\frac{\theta^{2}}{\pi} \mu_{n}^{2} \Delta m^{3}$

In addition, as far as the mirror photon is also massless, $n \rightarrow$ $n^{\prime} \gamma^{\prime}$ decay should take place with the same width, $\Gamma(n \rightarrow$ $\left.n^{\prime} \gamma^{\prime}\right)=\Gamma\left(n \rightarrow n^{\prime} \gamma\right)$ (once again, one can neglect the difference between the ordinary and mirror magnetic moments and take $\mu_{n}=\mu_{n^{\prime}}$ ). Thus, the total rate of $n \rightarrow n^{\prime}$ decay is $\Gamma\left(n \rightarrow n^{\prime}\right)=2 \theta^{2} \mu_{n}^{2} \Delta m^{3} / \pi=2 \mu_{n}^{2} \epsilon^{2} \Delta m / \pi$, with a photon $\gamma$ and mirror photon $\gamma^{\prime}$ channels both having equal ratios $=1 / 2$.

5. There can be additional decay channels with emission of some other massless bosons. Let us discuss the possibility when 
$n-n^{\prime}$ mixing emerges not at tree-level as in Fig. 2 but by loop mechanism shown in Fig. 3.

Let us assume that the heavy Dirac Fermions $N$ are not gauge singlets but are multiplets of some gauge group $S U\left(N_{C}\right)$ say in fundamental representations, $N^{a}$ and $N_{a}^{\prime}, a=1,2, \ldots N_{C}$ being the $S U\left(N_{C}\right)$ index, so that we have $N_{C}$ Dirac fermions with equal masses $M_{D} N^{a} N_{a}^{\prime}+$ h.c.. In this case, the Yukawa terms $S^{\dagger} d N$ and $S^{\prime \dagger} d^{\prime} N^{\prime}$ in (11) are forbidden by $S U\left(N_{C}\right)$ symmetry. However, one can introduce the additional colortriplet scalars $T^{a}$ and $T_{a}^{\prime}$ also in fundamental representations of $S U\left(N_{C}\right)$, and modify the Lagrangian terms (11) to the following:

$$
\begin{aligned}
& \mathcal{L}=S u d+S q q+T^{\dagger} d N+\text { h.c. } \\
& \mathcal{L}^{\prime}=S^{\prime} u^{\prime} d^{\prime}+S q^{\prime} q^{\prime}+T^{\prime \dagger} d^{\prime} N^{\prime}+\text { h.c. } \\
& \mathcal{L}_{\text {mix }}=S^{\dagger} S^{\prime \dagger} T T^{\prime}+M_{D} N N^{\prime}+\text { h.c. }
\end{aligned}
$$

In this case $n-n^{\prime}$ mixing is induced via the loop-diagram shown in Fig. 3 in which diagram would also induce the transitional moments $\kappa_{n n^{\prime}}$ and $\kappa_{n n^{\prime}}^{\prime}$ between the neutron and mirror neutron as in (9). One can imagine that there is also a gauge $U(1)$ symmetry in addition to $S U\left(N_{C}\right)$, with rather large coupling constant. In this way, in addition to ordinary and mirror photons, also a "third" photon $\gamma_{3}$ associated with the $U(1)$ gauge field $A_{\mu}^{(3)}$ enters the game. Then attaching the respective external photon line to the diagram of Fig. 3, one also obtains a transitional magnetic moment between $n$ and $n^{\prime}$ related to "third" photon, $\frac{1}{2} \kappa_{n n^{\prime}} F_{\mu v}^{(3)} \bar{n} \sigma^{\mu v} n^{\prime}+$ h.c. where $F_{\mu v}^{(3)}=$ $\partial_{\mu} A_{v}^{(3)}-\partial_{\nu} A_{\mu}^{(3)}$. In this way, there emerges an invisible decay channel $n^{\prime} \rightarrow n \gamma_{3}$ with a width

$\Gamma\left(n \rightarrow n^{\prime} \gamma_{3}\right)=\frac{x^{2}}{\pi} \mu_{n}^{2} \Delta m^{3}$

where $x=\kappa_{n n^{\prime}} / \mu_{n}$ is the "third" transitional magnetic moment in units of $\mu_{n}$. For large $N_{C}$, large gauge constant $g_{3}$ of extra $U(1)$, and large coupling constants in (14), $x$ can be comparable or even larger then $\theta$. In addition, the mass term induced by the loop can be suppressed by symmetry reasons making use of Voloshin's symmetry. In this way, the invisible decay channel $n^{\prime} \rightarrow n \gamma_{3}$ can become dominant. ${ }^{5}$

For total decay width of $n \rightarrow n^{\prime}$ decay we have

$$
\begin{aligned}
& \Gamma_{\text {new }}=\left(1+A_{\text {inv }}\right) \frac{\theta^{2}}{\pi} \mu_{n}^{2} \Delta m^{3}= \\
& \frac{1+A_{\text {inv }}}{6} \frac{\theta^{2}}{10^{-20}}\left(\frac{\Delta m}{1.57 \mathrm{MeV}}\right)^{3} \times 7 \cdot 10^{-30} \mathrm{GeV}
\end{aligned}
$$

where the decay width is normalized to the maximal mass difference $\Delta m_{\max }(5)$ allowed by ${ }^{9} \mathrm{Be}$ stability, and $A_{\text {inv }}$ denotes effective contribution of invisible decay channels as $n \rightarrow n^{\prime} \gamma^{\prime}$, $n \rightarrow n^{\prime} \gamma_{3}$ etc. which should be compared to the value (4) needed for explanation of the neutron lifetime discrepancy. The branching ratio of "yet-invisible" decay channel (with ordinary photon $\gamma)$ is

$\frac{\Gamma\left(n \rightarrow n^{\prime} \gamma\right)}{\Gamma_{\text {new }}}=\frac{1}{1+A_{\text {inv }}}$

${ }^{5}$ Yet another invisible decay channel can be $n \rightarrow n^{\prime}+\beta$ where $\beta$ is the Goldstone particle related to spontaneous breaking of $\mathrm{B}$ and $\mathrm{B}^{\prime}$ baryon numbers to a diagonal combination $\mathrm{B}+\mathrm{B}^{\prime}$ at some scale $V$ (implications of such Goldstones are discussed in Ref. [39]). This massless $\beta$ interacts between $n$ and $n^{\prime}$ states with the Yukawa coupling constant $g_{\beta}=\epsilon / V$, and thus $n \rightarrow n^{\prime} \beta$ decay rate can exceed that of $n \rightarrow n^{\prime} \gamma$ if $V<$ few $\mathrm{GeV}$.

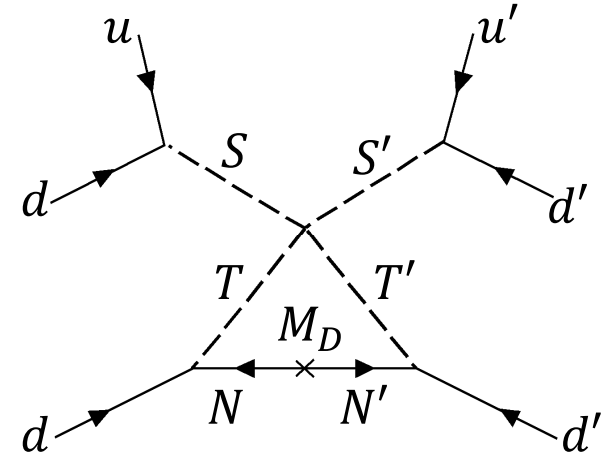

FIGURE 3: Loop diagram generating $n-n^{\prime}$ mixing through the strongly coupled $S U\left(N_{\mathcal{C}}\right)$ system

In particular, in the absence of "third" photon, and massless mirror photons coupled as ordinary one, i.e., $A_{\mathrm{inv}}=1$, we have $\operatorname{Br}\left(n \rightarrow n^{\prime} \gamma\right)=\operatorname{Br}\left(n \rightarrow n^{\prime} \gamma^{\prime}\right)=0.5$. If mirror and third photons are massive, we have $A_{\text {inv }}=0$ and only $n \rightarrow n^{\prime} \gamma$ remains. (e.g., due to $Z_{2}$-symmetry breaking, the VEVs of two doublets $\phi_{1,2}^{\prime}$ could break also mirror electric charge and thus render mirror photon massive). But for $x \gg \theta$ the invisible decay into third photon becomes large and the decay channel with ordinary photon becomes subdominant.

Solid curves in Fig. 4 show the parameter space (mixing angle $\theta$ vs. mirror neutron mass $m_{n^{\prime}}=m_{n}-\Delta m$ ) needed for achieving $\Gamma_{\text {new }}=7 \times 10^{-30} \mathrm{GeV}$ for different $A_{\text {inv }}$. Namely, the black solid curve corresponds to the case when $A_{\text {inv }}=0$, i.e., only $n \rightarrow n^{\prime} \gamma$ decay is operative: $\Gamma\left(n \rightarrow n^{\prime} \gamma\right) / \Gamma_{\text {new }}=1$. The solid purple corresponds to a benchmark case $A_{\text {inv }}=1$ when $n \rightarrow n^{\prime}$ decay occurs symmetrically with the ordinary and mirror photon emission, $\Gamma\left(n \rightarrow n^{\prime} \gamma\right)=\Gamma\left(n \rightarrow n^{\prime} \gamma^{\prime}\right)$. The brown and green curves show the cases when contribution of "third" photon $\gamma_{3}$ becomes dominant, respectively with $A_{\mathrm{inv}}=$ 3 and $A_{\text {inv }}=9$.

6. Let us discuss now implications of $n-n^{\prime}$ mixing and $n \rightarrow n^{\prime}$ decays provided that $m_{n^{\prime}}<m_{n}$ (but $m_{n^{\prime}}>m_{n}-\Delta m_{\max }=$ $937.99 \mathrm{MeV}$ as it is required by nuclear stability bound (5)) which crucially depend on the mass range of dark neutron $n^{\prime}$.

Namely, if $m_{n}^{\prime}>m_{p}+m_{e}=938.783 \mathrm{MeV}$, then mirror neutron $n^{\prime}$ (more precisely, the lighter mass eigenstate $n_{2}=$ $\left.n^{\prime}-\theta n\right)$ is not stable against $\beta$-decay $n^{\prime} \rightarrow p+e+\bar{v}_{e}$. Thus, the $\beta$-decay rates of $n$ and $n^{\prime}$ can be directly compared:

$$
\begin{aligned}
& \Gamma\left(n \rightarrow p e \bar{v}_{e}\right)=\frac{G_{V}^{2}\left(1+3 g_{A}^{2}\right) m_{e}^{5}}{2 \pi^{3}} F\left(\frac{Q}{m_{e}}\right) \\
& \Gamma\left(n^{\prime} \rightarrow p e \bar{v}_{e}\right)=\frac{\theta^{2} G_{V}^{2}\left(1+3 g_{A}^{2}\right) m_{e}^{5}}{2 \pi^{3}} F\left(\frac{Q^{\prime}}{m_{e}}\right)
\end{aligned}
$$

where $G_{V}=G_{F}\left|V_{u d}\right|$ is the Fermi constant corrected by Cabibbo mixing, $g_{A} \approx 1.27$ is the axial coupling constant, $Q=m_{n}-m_{p}-m_{e}=0.7823 \mathrm{MeV}$ and $Q^{\prime}=m_{n}^{\prime}-m_{p}+m_{e}<$ $0.7823 \mathrm{MeV}$ are respective $Q$-values. The function

$$
\begin{aligned}
& F(x)=\frac{\sqrt{x(x+2)}}{60}\left(2 x^{4}+8 x^{3}+3 x^{2}-10 x-15\right) \\
& +\frac{x+1}{4} \ln \left(1+x+\sqrt{x^{2}+2 x}\right)
\end{aligned}
$$

describes the phase space factor for the given $Q$-value. Therefore, the lifetime of $n^{\prime}$ can be related to the neutron lifetime and 


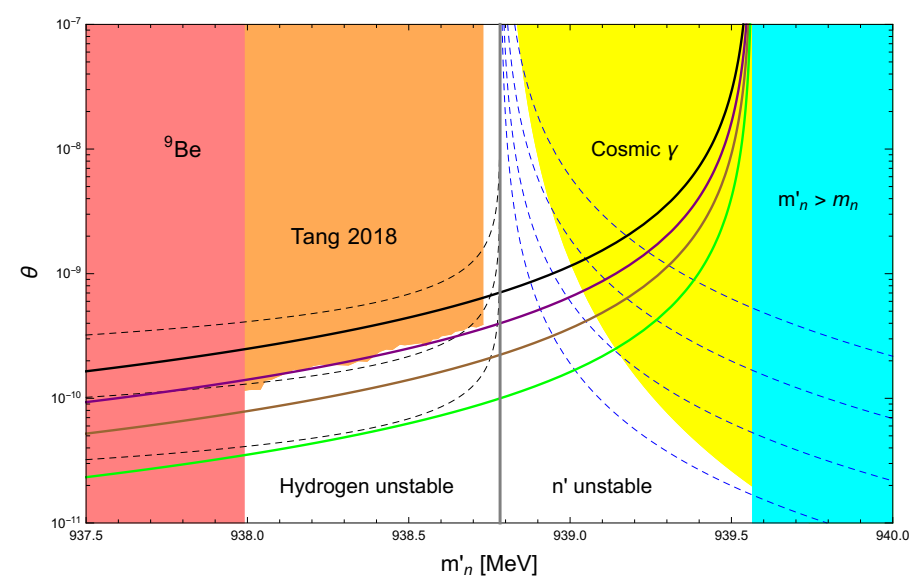

FIGURE 4: The allowed regions for the mirror neutron mass $n^{\prime}$ vs. $n-n^{\prime}$ mixing angle $\theta$. The black, purple, brown and green solid curves, all normalised to the needed decay width $\Gamma_{\text {new }}=$ $7 \times 10^{-30} \mathrm{GeV}$ (4), correspond respectively to the $n \rightarrow n^{\prime} \gamma$ branching ratios $\Gamma\left(n \rightarrow n^{\prime} \gamma\right) / \Gamma_{\text {new }}=1,0.5,0.25,0.1$. The vertical solid line separates regions $m_{n^{\prime}}>m_{p}+m_{e}$ (unstable dark neutron $n^{\prime}$ ) and $m_{n^{\prime}}<m_{p}+m_{e}$ (unstable hydrogen atom). The black dashed curves in the latter region correspond to ${ }^{1} \mathrm{H}$ lifetimes $\tau\left({ }^{1} \mathrm{H} \rightarrow n^{\prime} v_{e}\right)=10^{20}, 10^{21}$ and $10^{22} \mathrm{yr}$ (from up to down), while the blue dashed curves in the region $m_{n}^{\prime}>m_{p}+m_{e}$ correspond to $n^{\prime}$ lifetimes $\tau\left(n^{\prime} \rightarrow p e \bar{v}_{e}\right)=10^{14}, 10^{15}, 10^{16}$ and $10^{17}$ yr (again from up to down). The shaded regions are excluded by ${ }^{9}$ Be stability (pink), by limits on cosmic $\gamma$ background in the $0.1 \div 1 \mathrm{MeV}$ range (yellow), and by photon counting in the energy range of $780 \div 1600 \mathrm{keV}$ in recent experiment by Tang et al. (orange).

it can be estimated as

$$
\begin{aligned}
& \tau\left(n^{\prime} \rightarrow p e \bar{v}_{e}\right)=\frac{F\left(Q / m_{e}\right)}{F\left(Q^{\prime} / m_{e}\right)} \frac{\tau\left(n \rightarrow p e \bar{v}_{e}\right)}{\theta^{2}}= \\
& \frac{F\left(Q / m_{e}\right)}{F\left(Q^{\prime} / m_{e}\right)}\left(\frac{10^{-10}}{\theta}\right)^{2} \times 2.8 \times 10^{15} \mathrm{yr}
\end{aligned}
$$

The instability of dark matter is not a problem in itself once its decay time exceeds the age of the Universe $t_{U}=1.4 \times 10^{10} \mathrm{yr}$. In fact, a few percent fraction of dark matter decaying in invisible mode before present days could even help to reconcile the discrepancy between the Hubble constant value determined from the CMB measurements by Planck Satellite from one side, and its value obtained by direct astrophysical measurements from other side [40]. The problem is that $n^{\prime}$ decays into visible particles (proton and electron), together its radiative decay channel $n^{\prime} \rightarrow p e \bar{v}_{e} \gamma$ with a branching ratio $\sim 10^{-2}$, would contribute to cosmic diffuse $\gamma$ background at $\mathrm{MeV}$ energies.

The blue dash curves in Fig. 4 mark the parameter space which can lead to $n^{\prime}$ decay time in the range $10^{14}-10^{17} \mathrm{yr}$. Yellow shaded region corresponds to excluded region obtained by requiring, rather conservatively, that the $\gamma$ fluxes produced by these decays should not exceed their experimental values obtained by direct observations [41]. Taking into account that main contribution to $\gamma$-background in the $\mathrm{MeV}$ range is supposedly produced by the Seyfert galaxies and blazars, one should expect that real limits will be more stringent.

Dark neutron $n^{\prime}$ would be stable if its mass is small enough. Namely, if $m_{n^{\prime}}<m_{p}+m_{e}=938.783 \mathrm{MeV}$, the decay $n^{\prime} \rightarrow p e \bar{v}_{e}$ is forbidden and $n^{\prime}$ would be a stable dark particle. However, this situation would imply that the hydrogen atom ${ }^{1} \mathrm{H}$ (protium) should be unstable, and it would decay into dark neutron and ordinary neutrino via electron capture, $p+e \rightarrow n^{\prime}+v_{e}$. Its decay width can be readily estimated as ${ }^{6}$

$$
\begin{aligned}
& \Gamma\left({ }^{1} \mathrm{H} \rightarrow n^{\prime} v_{e}\right)=\frac{\theta^{2} G_{V}^{2}\left(1+3 g_{A}^{2}\right)}{2 \pi^{2} a_{0}^{3}}\left(m_{p}+m_{e}-m_{n}^{\prime}\right)^{2} \\
& =\theta^{2}\left(\frac{m_{p}+m_{e}-m_{n^{\prime}}}{0.783 \mathrm{MeV}}\right)^{2} \times 1.23 \cdot 10^{-33} \mathrm{GeV}
\end{aligned}
$$

where $a_{0}=\left(\alpha m_{e}\right)^{-1}$ is the Bohr radius. Thefore, for the lifetime of the hydrogen atom we get

$\tau\left({ }^{1} \mathrm{H}\right)=\frac{17 \mathrm{yr}}{\theta^{2}}\left(\frac{0.783 \mathrm{MeV}}{m_{p}+m_{e}-m_{n^{\prime}}}\right)^{2}$

Black dashed curves in Fig. 4 correspond to protium lifetimes in the range of $10^{20}-10^{22} \mathrm{yr}$.

Surprisingly, no direct experimental limits are available on the protium dark decay into a dark particle $n^{\prime}$ and (in practice invisible) neutrino. Very existence of our universe limits the hydrogen decay time to be larger than the present cosmological age $t_{u}=1.4 \times 10^{10} \mathrm{yr}$. Disappearance of more than $1 \%$ of the hydrogen in the Universe would affect the Big Bang Nucleosynthesis tests. Thus, from the primordial hydrogen abundance one can infer a conservative lower bound $\tau\left({ }^{1} \mathrm{H} \rightarrow\right.$ inv $)>1.4 \times 10^{12}$ yr or so.

Somewhat stronger bounds can be obtained from the electron capture process $p+e \rightarrow n^{\prime}+v_{e}$ in the sun which produces mono-energetic neutrinos with $E_{v} \approx m_{p}+m_{e}-m_{n^{\prime}}$. Let us take the maximal value $E=0.78 \mathrm{MeV}$ which corresponds to the lower extreme $m_{n^{\prime}}=937.992 \mathrm{MeV}$ in (5). Let us assume that flux $\phi_{n^{\prime}}^{v}$ of $v_{e}$ produced in this way is no larger than one fifth of the ${ }^{7}$ Be solar neutrino flux $\phi_{\mathrm{Be}}^{v} \simeq 5 \times 10^{9} \mathrm{~cm}^{-2} \mathrm{~s}^{-1}$ $\left(E_{\mathrm{Be}}^{v}=0.862 \mathrm{MeV}\right)$ - otherwise it would be detected by BOREXINO experiment (this assumption on the BOREXINO sensitivity is of course is an exaggeration). In other words, we infer that $\phi_{n^{\prime}}^{v}<10^{9} \mathrm{~cm}^{-2} \mathrm{~s}^{-1}$ which puts the lower limit on the proton lifetime $\tau_{p e}$ against the electron capture process $p e \rightarrow n^{\prime} v_{e}$ in the sun, $\tau_{p e}>7 \times 10^{11} \mathrm{yr}$ or so. For the sake of comparison, the proton lifetime against the dominant reaction $p p \rightarrow d e^{+} v_{e}$, alias the hydrogen burning time in the sun, is $t_{p p} \simeq 1.2 \times 10^{10} \mathrm{yr}$, i.e., practically the age of the Universe.

The rate of $p+e \rightarrow n^{\prime}+v_{e}$ reaction in the sun can be obtained from the hydrogen atom decay rate (21) roughly by substituting the electron density in atom $1 / a_{0}^{3}$ by the electron density in solar plasma $n_{e}=\rho X / m_{p}$. More precise calculation of the unbound electron capture rate, obtained by integration over the Maxwell-Boltzmann distribution of electrons, gives

$$
\begin{aligned}
& \frac{\Gamma\left(p e \rightarrow n^{\prime} v_{e}\right)}{\Gamma\left({ }^{1} \mathrm{H} \rightarrow n^{\prime} v_{e}\right)}=\sqrt{\frac{2 \pi^{3} m_{e}}{T}} \alpha\left(n_{e} a_{0}^{3}\right) \\
& \simeq 30 \times\left(\frac{10^{6} \mathrm{~K}}{T}\right)^{1 / 2}\left(\frac{n_{e}}{10^{24} / \mathrm{cm}^{3}}\right)
\end{aligned}
$$

Taking typical temperature as $T \simeq 10^{7} \mathrm{~K}$, typical density $\rho \simeq 100 \mathrm{~g} / \mathrm{cm}^{3}$ and the hydrogen mass fraction $X \simeq 0.5$ and

${ }^{6}$ It should be stressed that we talk about the hydrogen atom and not its nucleus (proton). In fact, the proton decay $p \rightarrow n^{\prime} e^{+} v_{e}$ would occur if $m_{n^{\prime}}<m_{p}-m_{e}=$ 937.761 MeV which is already excluded by ${ }^{9}$ Be stability, $m_{n^{\prime}}>937.992 \mathrm{MeV}$ (5). 
thus $n_{e} \simeq 25 \times 10^{24} \mathrm{~cm}^{-3}$ in solar interior within the radius $0.2 R_{\odot}$, we get $\Gamma\left(p e \rightarrow n^{\prime} v_{e}\right) / \Gamma\left({ }^{1} \mathrm{H} \rightarrow n^{\prime} v_{e}\right) \simeq 250$. Therefore, the bound $\tau_{p e}>7 \times 10^{11} \mathrm{yr}$ inferred for mono-energetic neutrinos with $E_{v}=0.78 \mathrm{MeV}$ is roughly equivalent to $\tau\left({ }^{1} \mathrm{H}\right)>$ $2 \times 10^{14} \mathrm{yr}$ or so. Applying the same consideration for smaller values of $E_{v}=m_{p}+m_{e}-m_{n^{\prime}}$, e.g., $E_{v}=0.4 \mathrm{MeV}$, the $v_{e}$ flux produced by $p+e \rightarrow n^{\prime}+v_{e}$ process should be compared with the dominant $p p$ neutrino flux, which would render this limit more than an order of magnitude weaker, around $10^{13} \mathrm{yr}$.

On the other hand, the reaction $p+e \rightarrow n^{\prime}+v_{e}$ produces dark fermions $n^{\prime}$ right inside the sun. Their presence, if abundant, would change the thermal conductivity of solar interior and this be tested by the helio-seismological data which requires a specific study. By inferring that the overall mass of $n^{\prime}$ produced in the sun from its birth is smaller than e.g., $10^{-6} M_{\odot}$, we would get $t_{p e}>10^{15} \mathrm{yr}$ or so which translates into $\tau\left({ }^{1} \mathrm{H}\right)>2.5 \times 10^{17} \mathrm{yr}$. Thus, it seems that the solar physics cannot exclude the protium lifetime as large as $\tau\left({ }^{1} \mathrm{H}\right)=10^{21} \mathrm{yr}$ typical for our model.

One can discuss also the electron capture processes by nuclei $(Z, A)+e \rightarrow(Z-1, A-1)+n^{\prime}+v_{e}$ in the electrondegenerate cores of the heavier stars. Let us consider carbon white dwarfs with a central densities $\rho \sim 10^{6} \mathrm{~g} / \mathrm{cm}^{3}$ at which densities electrons become relativistic, i.e., their Fermi momentum $p_{F} \approx 3.1 n_{e}^{1 / 3}$ becomes comparable to the electron mass $m_{e}$. However, it is not enough to overcome the energy threshold of ${ }^{12} \mathrm{C}+e \rightarrow{ }^{11} \mathrm{~B}+n^{\prime}+v_{e}$ reaction which is above $15 \mathrm{MeV}$. The same applies to the case of oxygen, ${ }^{16} \mathrm{O}+e \rightarrow{ }^{15} \mathrm{~N}+n^{\prime}+v_{e}$ which has threshold energy of about $12 \mathrm{MeV}$. Therefore, such processes can start only at densities approaching $10^{9} \mathrm{~g} / \mathrm{cm}^{3}$, as e.g. in heavy white dwarfs with masses saturating Chandrasekhar limit or in dense pre-supernova cores of very massive stars. The question whether this can have any observable consequences or whether it can set stronger limit on the hydrogen lifetime deserves special consideration.

A "silent" disappearance of hydrogen atom ${ }^{1} \mathrm{H} \rightarrow n^{\prime} v_{e}$ leaving the party without saying "Good Bye" is difficult to detect experimentally. Even the daughter radiative branch ${ }^{1} \mathrm{H} \rightarrow$ $n^{\prime} v_{e} \gamma$, with emission of a single photon with the energy up to $0.78 \mathrm{MeV}$, can be hardly discriminated from the background. The intriguing possibility that hydrogen, the most abundant chemical element in the Universe constituting about $75 \%$ of its visible mass, can in fact be metastable remains as a challenge for future experiments.

In principle, some other elements $(Z, A)$ could also decay via the electron capture ep $\rightarrow n^{\prime} v_{e}$. However, for any stable element (apart the hydrogen) the ${ }^{9}$ Be stability condition (5) does not leave an available phase space for the transition $(Z, A) \rightarrow(Z-1, A-1)+n^{\prime}+v_{e}$. Perhaps it would be interesting to address exotic decays of some unstable proton-rich elements for which such transitions are allowed. ${ }^{7}$

Consider for example, ${ }^{48} \mathrm{Ni}$ which has a doubly-magic nuclei $(Z=28, A=48)$, and decay time $2.1 \mathrm{~ms}$. There is no bound nuclei $(Z=27, A=48)$ to which ${ }^{48} \mathrm{Ni}$ could transform via $\beta^{+}$-decay or electron capture, and there is no bound nuclei

${ }^{7}$ Let us remark that at the lower edge of allowed range (5), namely for $m_{n^{\prime}}<$ $938.06 \mathrm{MeV}$, the deuterium atom decay ${ }^{2} \mathrm{H} \rightarrow 2 n^{\prime}+v_{e}+X$ becomes cinematically allowed, as a combination of electron capture $p+e \rightarrow n^{\prime}+v_{e}$ and neutron decay $n \rightarrow n^{\prime}+X$. However, due to a minuscule phase space and double suppression $\sim$ $\theta^{4}$, the deuterium lifetime will be extremely large, beyond any practical interest. For any other stable element even such a double decay is cinematically forbidden.
$(Z=27, A=47)$ to which it can transform by expelling one proton. In fact, ${ }^{48} \mathrm{Ni}$ can decay via double processes, electron capture by one proton accompanied by $\beta^{+}$-decay of another proton, ${ }^{48} \mathrm{Ni} \rightarrow{ }^{48} \mathrm{Fe}(Z=26, A=48)$ or via expelling simultaneously two protons, ${ }^{48} \mathrm{Ni} \rightarrow{ }^{46} \mathrm{Fe}+2 p e(Z=26, A=46)$. The electron capture $e p \rightarrow n v_{e}$ with outflowing neutron and simultaneous expelling of proton ${ }^{48} \mathrm{Ni} \rightarrow{ }^{46} \mathrm{Fe}+n+p+e$ is marginally allowed by phase space but it is suppressed kinematically since $m_{n}>m_{p}+m_{e}$. However, in the case of $m_{n^{\prime}}<$ $m_{p}+m_{e}$ such transition with emission of dark neutron $n^{\prime}$ will not be suppressed and thus ${ }^{48} \mathrm{Ni} \rightarrow{ }^{46} \mathrm{Fe}$ transition without ordinary neutron $n$ could take place at detectable level accompanied by only one proton. Unfortunately, not much is known about ${ }^{48} \mathrm{Ni}$ decay channels. Some other elements also can be of interest. For example, ${ }^{50} \mathrm{Co}\left(T_{1 / 2}=38.8 \mathrm{~ms}\right)$ via electron capture $e p \rightarrow n^{\prime} v_{e}$ could be transformed into ${ }^{49} \mathrm{Fe}$, if $m_{n^{\prime}}<938.615$ $\mathrm{MeV}$. Analogously ${ }^{12} \mathrm{~N}\left(T_{1 / 2}=11 \mathrm{~ms}\right)$, apart of its usual $\beta^{+}$ decay into the stable ${ }^{12} \mathrm{C}$, would have a new decay channel ${ }^{12} \mathrm{~N} \rightarrow{ }^{11} \mathrm{C}+n^{\prime}+v_{e}$ if $m_{n^{\prime}}<938.182 \mathrm{MeV}$, with easily detectable ${ }^{11} \mathrm{C}\left(T_{1 / 2}=20 \mathrm{~m}\right)$. Another example, relatively stable ${ }^{8} \mathrm{~B}\left(T_{1 / 2}=770 \mathrm{~ms}\right)$ has usual $\beta^{+}$decay into ${ }^{8} \mathrm{Be}$ which then promptly decays in two $\alpha$-particles. In the case, $m_{n^{\prime}}<938.646$ $\mathrm{MeV}$, it could have a decay channel into ${ }^{7} \mathrm{Be}$. Via the electron capture, the latter would end up in ${ }^{7} \mathrm{Li}$ after 53 days or so.

Concluding this section, provided that the beryllium bound (5) is fulfilled, $n \rightarrow n^{\prime}$ decay has no strong observable consequences for nuclei besides the intriguing possibility that the hydrogen atom can be unstable. However, it will have dramatic consequences for neutron stars (NS). Given that the equation of state (EoS) of mirror nuclear matter, despite a $\mathrm{MeV}$ range mass difference between ordinary and mirror nucleons, should be essentially the same, $n \rightarrow n^{\prime}$ conversion would rapidly transform the ordinary NS, after its birth, into a mixed star with half of its mass constituted by mirror matter. Now two components with the same EoS can be "packed" inside the same volume which changes the pressure - mass balance in the star and thus changes the mass-radius relations. Namely, the mixed NS will be more compact than the initial pure neutron star of the given mass. with the radius of about a factor of $\sqrt{2}$ smaller than the initial radius of the newborn NS. On the other hand, also the maximal mass of the mixed NS will be reduced by a factor of $\sqrt{2}$ with respect of the pure NS. For example, a realistic EoS of Ref. [42] for pure NS can support $M_{\max } \simeq 2.1 M_{\odot}$, in which case maximal mass of mixed NS is about $1.5 \mathrm{M}_{\odot}$; any NS with a larger mass should collapse to black hole. Therefore, NS of $2 M_{\odot}$ could not exist unless the EoS is so stiff that can support $M_{\max } \simeq 3 M_{\odot}$ for a pure NS. One can consider also a possibility that after the supernova explosion the newly born NS suffers a matter infall, its mass rapidly reaches a critical value and within days it transforms into a quark star dominantly composed of deconfined quark matter, which could also explain the events of delayed GRB events correlated with the supernova explosions [43]. In this case, the observed NS with larger masses reaching $2 M_{\odot}$ can be considered as quark stars. The implications of $n \rightarrow n^{\prime}$ transition will be addressed in more details elsewhere [44].

7. The suggested scenario implies that the neutron has two decay channels, $\beta$-decay and hypothetical invisible decay. Therefore, the beam experiments measure its $\beta$-decay width $\Gamma_{\beta}=$ $\tau_{\text {beam, }}^{-1}$ while trap experiments measure the total decay width, $\Gamma_{n}=\tau_{\text {trap }}^{-1}$. However, one can question whether this hypoth- 
esis is compatible with other precision measurements regarding the determination of the Fermi constant $G_{F}$, the CKM mixing element $V_{u d}$ and the ratio of axial and vector constants $g_{A}=-G_{A} / G_{V}$.

The neutron $\beta$-decay $n \rightarrow p e \bar{v}_{e}$ is described by the Fermi Lagrangian

$\frac{G_{V}}{\sqrt{2}} \bar{p} \gamma^{\mu}\left(1-g_{A} \gamma^{5}\right) n \bar{e} \gamma_{\mu}\left(1-\gamma^{5}\right) v_{e}$

where $g_{A}$ is the axial coupling constant. In the context of the Standard Model, we have $G_{V}=G_{F}\left|V_{u d}\right|$, where $V_{u d}$ is the CKM mixing element. Then, for $G_{F}$ determined from the muon decay, i.e., $G_{F}=G_{\mu}=1.1663787(6) \times 10^{-5} \mathrm{GeV}^{-2}$ [21], the neutron $\beta$-decay lifetime $\tau_{\beta}$ is given by the well-known formula $\tau_{n}\left|V_{u d}\right|^{2}\left(1+3 g_{A}^{2}\right)=(4908.7 \pm 1.9) \mathrm{s}$ which includes Coulomb corrections as well as external radiative corrections [45]. In more generic form, having in mind possible effects of new physics beyond Standard Model and without assuming $G_{V}=G_{\mu}\left|V_{u d}\right|$, it can be presented as

$\tau_{\beta}=\frac{(4908.7 \pm 1.9) \mathrm{s}}{\left(G_{V} / G_{\mu}\right)^{2}\left(1+3 g_{A}^{2}\right)}$

The constant $G_{V}$ is experimentally measured by the study of super-allowed $0^{+} \rightarrow 0^{+}$nuclear $\beta$-decays which are pure vector transitions, modulo theoretical uncertainties due to nuclear Coulomb effects and radiative corrections. For $G_{F}=G_{\mu}$, these measurements yield the world average $\left|V_{u d}\right|=0.97417 \pm$ 0.00021 [20] which value is also well-compatible with the unitarity of the CKM mixing matrix. In more general BSM context, without taking $G_{V}=G_{\mu}\left|V_{u d}\right|$, we have:

$\left(G_{V} / G_{\mu}\right)^{2}=0.94901 \pm 0.00041$

Therefore, Eq. (25) can be presented as

$\tau_{\beta}=\frac{(5172.5 \pm 2.0) \mathrm{s}}{1+3 g_{A}^{2}}$

where the value (26) is substituted for $\left(G_{V} / G_{\mu}\right)^{2}$.

The axial coupling constant $g_{A}$ involving non-perturbative contributions is poorly determined theoretically. But it is well determined experimentally via measuring $\beta$-asymmetries. The world average $g_{A}=1.2723 \pm 0.0023$ reported in PDG [20] then implies $\tau_{n}=883.2 \pm 3.0 \mathrm{~s}$, compatible with both trap and beam within $1 \sigma$. However, the average value obtained by last two most precise experiments $[46,47]$ imply higher value $g_{A}=1.2764 \pm 0.0013$ gives $\tau_{n}=878.5 \pm 1.9 \mathrm{~s}$ which is consistent with $\tau_{\text {trap }}$ but it is in tension with $\tau_{\text {beam }}$. In fact, this tension between the latest results on $g_{A}$ measurements $[46,47]$ and the neutron beam lifetime $\tau_{\text {beam }}$ is clearly demonstrated in Fig. 6 of Ref. [47]. If the forthcoming experiments on $\beta$-asymmetries will confirm these results on $g_{A}$, and thus increase the tension with the value of $\tau_{\beta}$ determined by the beam measurements, then the neutron dark decay $n \rightarrow n^{\prime} X$ will become useless for understanding the neutron lifetime anomaly. In fact, a new upper limits on the $n \rightarrow n^{\prime} X$ decay rate about ten times stronger than (4), at the level $\Gamma_{\text {new }}<10^{-30} \mathrm{GeV}$ can be firmly established, nearly corresponding to the green curve in Fig. 4 . However, the possibility of exotic neutron decay may have independent interest, also having in mind its intriguing implication for the hydrogen atom instability. In fact, as one can see from Fig. 4, this limit would imply the hydrogen atom lifetime of about $10^{22} \mathrm{yr}$.

\section{Acknowledgements}

This work is mainly based on my talk reported at the Workshop INT-17-69W "Neutron-Antineutron Oscillations: Appearance, Disappearance, and Baryogenesis", Seattle, USA, October $23-27,2017$ and the slides are available at [48]. I would like to thank the Institute of Nuclear Theory for hospitality in Seattle. I also thank Pierluigi Belli, Yuri Kamyshkov, Klaus Kirch, ChenYu Liu, David McKeen, Rabi Mohapatra, Valery Nesvizhevsky, Arkady Vainshtein, Albert Young and other participants of the Workshop for interesting discussions and comments. Main part of this paper including Figures was practically written between November 2017 and January 2018. I thank Riccardo Biondi and Yuri Kamyshkov for helping in preparation of Figures. However, I did not rush to post this work to arXiv since in December 2017 the UCNA collaboration [47] reported new results on $\beta$-asymmetry measurements in the neutron decay. The new value of the axial coupling constant $g_{A}$, determined in combination with the previous PERKEO II result [46], is inconsistent with the hypothesis that the neutron lifetime discrepancy is due to the neutron invisible decay. Therefore, I revisited the manuscript adding last section, fixed some typos and added new references, and also presented this work at the NNBAR at ESS Workshop No. 8, 22-23 May 2018, Institut Laue-Langevin, Grenoble [49].

After my Seattle talk [48] a similar work of Fornal and Grinstein appeared [50], with the difference that the dark particle $n^{\prime}$ was considered as an elementary fermion with a mass chosen ad hoc. It was followed by other works [51, 52, 53, 54, 55, 56, $57,58,59,60,61]$ which essentially excluded the version with elementary $n^{\prime}$ from the neutron star stability [54, 55, 56, 57], and excluded $n \rightarrow n^{\prime} \gamma$ decay with 1 percent branching ratio [51]. In addition, Ref. [52] showed that the dark decay solution generically suffers from $g_{A}$-inconsistency problem. Evidently, my talk [48] had some subconscious impact on the community. It is somewhat surprising that non of these works mentioned about it though some authors of Refs. [51, 54] were present at my talk in Seattle and some others were informed about it.

I was uncertain about publishing this work, mainly because of understanding that the neutron dark decay solution is incompatible with the value of axial coupling $g_{A}$. In addition, for a while I found $g_{A}$ consistent solution to the neutron lifetime puzzle based on $n-n^{\prime}$ oscillation [62]. Finally I decided that the present work can be anyway published, at least for the intriguing possibility of the hydrogen metastability which was overlooked in previous papers. Perhaps the exotic decays of the neutron and hydrogen may work in some future (one never knows) towards understanding the fundamental physics behind the neutron.

\section{References}

[1] A. P. Serebrov and A. K. Fomin, Physics Procedia 17, 199 (2011) [arXiv:1104.4238 [nucl-ex]].

[2] F. E. Wietfeldt, arXiv:1411.3687 [nucl-ex]; G. L. Greene and P. Geltenbort, Scientific American 314, 36 (2016).

[3] A. K. Kharitonov et al., Nucl. Instrum. Meth. A 284, 98 (1989).

[4] W. Paul et al., Z. Phys. C 45, 25 (1989).

[5] W. Mampe et al., JETP Lett. 57, 82 (1993) [Pisma Zh. Eksp. Teor. Fiz. 57, 77 (1993)]. 
[6] A. Serebrov et al., Phys. Lett. B 605, 72 (2005) [nuclex/0408009]; see also A. P. Serebrov et al., Phys. Rev. C 78, 035505 (2008) [nucl-ex/0702009].

[7] A. Pichlmaier, V. Varlamov, K. Schreckenbach and P. Geltenbort, Phys. Lett. B 693, 221 (2010).

[8] A. Steyerl et al., Phys. Rev. C 85, 065503 (2012); revaluated results of MAMBO I experiment W. Mampe et al., Phys. Rev. Lett. 63, 593 (1989).

[9] S. S. Arzumanov et al., JETP Lett. 95, 224 (2012) [Pisma Zh. Eksp. Teor. Fiz. 95, 248 (2012)]; revaluated results of S. Arzumanov et al., Phys. Lett. B 483, 15 (2000).

[10] V. F. Ezhov et al., JETP Lett. 107, no. 11, 671 (2018) [arXiv:1412.7434 [nucl-ex]].

[11] S. Arzumanov et al., Phys. Lett. B 745, 79 (2015).

[12] R. W. Pattie, Jr. et al., Science 360, no. 6389, 627 (2018) [arXiv:1707.01817 [nucl-ex]].

[13] A. P. Serebrov et al., Phys. Rev. C 97, no. 5, 055503 (2018) [arXiv:1712.05663 [nucl-ex]].

[14] P. E. Spivak, Sov. Phys. JETP 67, 1735 (1988).

[15] J. Byrne et al., Europhys. Lett. 33, 187 (1996); revaluated results of Phys. Rev. Lett. 65, 289 (1990).

[16] A. T. Yue et al., Phys. Rev. Lett. 111, no. 22, 222501 (2013) [arXiv:1309.2623 [nucl-ex]]; revaluated results of J. S. Nico et al., Phys. Rev. C 71, 055502 (2005) [nucl-ex/0411041]; M. S. Dewey et al., Phys. Rev. Lett. 91, 152302 (2003) [nuclex/0311006].

[17] K. Nakamura et al. [Particle Data Group], J. Phys. G 37, 075021 (2010).

[18] J. Beringer et al. [Particle Data Group], Phys. Rev. D 86, 010001 (2012).

[19] K. A. Olive et al. [Particle Data Group], Chin. Phys. C 38, 090001 (2014).

[20] C. Patrignani et al. [Particle Data Group], Chin. Phys. C 40, no. 10, 100001 (2016).

[21] M. Tanabashi et al. [Particle Data Group], Phys. Rev. D 98, no. 3, 030001 (2018).

[22] A. P. Serebrov and A. K. Fomin, Phys. Rev. C 82, 035501 (2010); [arXiv:1005.4312 [nucl-ex]]; JETP Lett. 92, 40 (2010) [arXiv:1005.2906 [nucl-ex]].

[23] G. Narain, J. Schaffner-Bielich and I. N. Mishustin, Phys. Rev. D 74, 063003 (2006) [astro-ph/0605724].

[24] J. R. Oppenheimer and G. M. Volkoff, Phys. Rev. 55, 374 (1939).

[25] S. L. Shapiro and S. A. Teukolsky, "Black Holes, White Dwarfs and Neutron Stars: The Physics of Compact Objects", Wiley, 1983

[26] Z. Berezhiani, Int. J. Mod. Phys. A 19, 3775 (2004) [hepph/0312335]; "Through the looking-glass: Alice's adventures in mirror world," In From Fields to Strings, Circumnavigating Theoretical Physics, Eds. M. Shifman et al., vol. 3, pp. 2147-2195 [hep-ph/0508233].

[27] Z. Berezhiani, Eur. Phys. J. ST 163, 271 (2008); R. Foot, Int. J. Mod. Phys. A 29, 1430013 (2014). For a concise historical overview and related references see also L. B. Okun, Phys. Usp. 50, 380 (2007) [hep-ph/0606202].

[28] Z. Berezhiani, A. D. Dolgov and R. N. Mohapatra, Phys. Lett. B 375, 26 (1996) [hep-ph/9511221]; Z. Berezhiani, Acta Phys. Polon. B 27, 1503 (1996) [hep-ph/9602326]; R. N. Mohapatra and V. L. Teplitz, Astrophys. J. 478, 29 (1997) [astro-ph/9603049]; Z. Berezhiani, L. Gianfagna and M. Giannotti, Phys. Lett. B 500, 286 (2001) [hepph/0009290].
[29] Z. Berezhiani, D. Comelli and F. L. Villante, Phys. Lett. B 503, 362 (2001) [hep-ph/0008105].

[30] A. Y. Ignatiev and R. R. Volkas, Phys. Rev. D 68, 023518 (2003); [hep-ph/0304260]; Z. Berezhiani, P. Ciarcelluti, D. Comelli and F. L. Villante, Int. J. Mod. Phys. D 14, 107 (2005) [astro-ph/0312605]; Z. Berezhiani, S. Cassisi, P. Ciarcelluti and A. Pietrinferni, Astropart. Phys. 24, 495 (2006) [astro-ph/0507153]; S. J. Lonsdale and R. R. Volkas, Phys. Rev. D 97, 103510 (2018) [arXiv:1801.05561 [hep-ph]].

[31] L. Bento and Z. Berezhiani, Phys. Rev. Lett. 87, 231304 (2001) [hep-ph/0107281]; Fortsch. Phys. 50, 489 (2002) [hep-ph/0111116]; Z. Berezhiani, Nucl. Phys. Proc. Suppl. 237, 263 (2013); arXiv:1602.08599 [astro-ph.CO].

[32] E. K. Akhmedov, Z. Berezhiani and G. Senjanovic, Phys. Rev. Lett. 69, 3013 (1992) [hep-ph/9205230]; R. Foot, H. Lew and R. R. Volkas, Mod. Phys. Lett. A 7, 2567 (1992); R. Foot and R. R. Volkas, Phys. Rev. D 52, 6595 (1995) [hepph/9505359]; Z. Berezhiani and R. N. Mohapatra, Phys. Rev. D 52, 6607 (1995) [hep-ph/9505385].

[33] Z. Berezhiani and L. Bento, Phys. Rev. Lett. 96, 081801 (2006) [hep-ph/0507031]; Phys. Lett. B 635, 253 (2006) [hep-ph/0602227];

[34] R. N. Mohapatra, S. Nasri and S. Nussinov, Phys. Lett. B 627, 124 (2005) [hep-ph/0508109]; Z. Berezhiani and A. Gazizov, Eur. Phys. J. C 72, 2111 (2012) [arXiv:1109.3725 [astro-ph.HE]]; A. Coc, J. P. Uzan and E. Vangioni, Phys. Rev. D 87, no. 12, 123530 (2013) [arXiv:1303.1935 [astroph.CO]]; A. Addazi, Z. Berezhiani and Y. Kamyshkov, Eur. Phys. J. C 77, no. 5, 301 (2017) [arXiv:1607.00348 [hep-ph]]; R. N. Mohapatra and S. Nussinov, Phys. Lett. B 776, 22 (2018) [arXiv:1709.01637 [hep-ph]].

[35] D. G. Phillips, II et al., Phys. Rept. 612, 1 (2016); K. Babu et al., arXiv:1310.8593 [hep-ex].

[36] Y. N. Pokotilovski, Phys. Lett. B 639, 214 (2006) [nuclex/0601017]; Z. Berezhiani, Eur. Phys. J. C 64, 421 (2009) [arXiv:0804.2088 [hep-ph]]; Z. Berezhiani, M. Frost, Y. Kamyshkov, B. Rybolt and L. Varriano, Phys. Rev. D 96, no. 3, 035039 (2017) [arXiv:1703.06735 [hep-ex]]; L. Broussard et al., arXiv:1710.00767 [hep-ex].

[37] G. Ban et al., Phys. Rev. Lett. 99, 161603 (2007) [arXiv:0705.2336 [nucl-ex]]; A. Serebrov et al., Phys. Lett. B 663, 181 (2008) [arXiv:0706.3600 [nucl-ex]]; K. Bodek et al., Nucl. Instrum. Meth. A 611, 141 (2009); A. Serebrov et al., Nucl. Instrum. Meth. A 611, 137 (2009) [arXiv:0809.4902 [nucl-ex]]; I. Altarev et al., Phys. Rev. D 80, 032003 (2009) [arXiv:0905.4208 [nucl-ex]]; Z. Berezhiani and F. Nesti, Eur. Phys. J. C 72, 1974 (2012) [arXiv:1203.1035 [hep-ph]]. Z. Berezhiani et al., Eur. Phys. J. C 78, no. 9, 717 (2018) [arXiv:1712.05761 [hep-ex]].

[38] Z. Berezhiani and A. Vainshtein, Phys. Lett. B 788, 58 (2019) [arXiv:1809.00997 [hep-ph]]; Int. J. Mod. Phys. A 33, no. 31, 1844016 (2018) [arXiv:1506.05096 [hep-ph]]; see also Z. Berezhiani, R. Biondi, Y. Kamyshkov and L. Varriano, arXiv:1812.11141 [nucl-th].

[39] Z. Berezhiani, Eur. Phys. J. C 76, no. 12, 705 (2016) [arXiv:1507.05478 [hep-ph]];

[40] Z. Berezhiani, A. D. Dolgov and I. I. Tkachev, Phys. Rev. D 92, no. 6, 061303 (2015) [arXiv:1505.03644 [astro-ph.CO]].

[41] Y. Inoue, "Cosmic Gamma-ray Background Radiation," arXiv:1412.3886 [astro-ph.HE].

[42] F. Douchin and P. Haensel, Astron. Astrophys. 380, 151 (2001) [astro-ph/0111092]. 
[43] Z. Berezhiani, I. Bombaci, A. Drago, F. Frontera and A. Lavagno, Astrophys. J. 586, 1250 (2003) [astro-ph/ 0209257].

[44] Z. Berezhiani, R. Biondi, M. Mannarelli and F. Tonelli, in preparation

[45] W. J. Marciano and A. Sirlin, Phys. Rev. Lett. 96, 032002 (2006) [hep-ph/0510099].

[46] D. Mund et al., Phys. Rev. Lett. 110, 172502 (2013) [arXiv:1204.0013 [hep-ex]].

[47] M. A.-P. Brown et al. [UCNA Collaboration], Phys. Rev. C 97, no. 3, 035505 (2018) [arXiv:1712.00884 [nucl-ex]].

[48] Z. Berezhiani, "Unusual effects in n-n' conversion", talk at INT Workshop INT-17-69W, Seattle, 23-27 Oct. 2017, http://www.int. washington.edu/talks/WorkShops/ int_17_69W/People/Berezhiani_Z/Berezhiani3.pdf

[49] Z. Berezhiani, "Unusual effects in $n-n$ ' conversion", NNBAR at ESS workshop 8, Institut LaueLangevin, Grenoble, France, 22-23 May 2018 https:// indico.esss.lu.se/event/1002/contributions/ 36/attachments/44/69/Berezhiani-2018-ILL_ESS.pdf

[50] B. Fornal and B. Grinstein, Phys. Rev. Lett. 120, no. 19, 191801 (2018) [arXiv:1801.01124 [hep-ph]].
[51] Z. Tang et al., Phys. Rev. Lett. 121, no. 2, 022505 (2018) [arXiv:1802.01595 [nucl-ex]].

[52] A. Czarnecki, W. J. Marciano and A. Sirlin, Phys. Rev. Lett. 120, no. 20, 202002 (2018) [arXiv:1802.01804 [hep-ph]].

[53] A. P. Serebrov, R. M. Samoilov, I. A. Mitropolsky and A. M. Gagarsky, arXiv:1802.06277 [nucl-ex].

[54] D. McKeen, A. E. Nelson, S. Reddy and D. Zhou, Phys. Rev. Lett. 121, 061802 (2018) [arXiv:1802.08244 [hep-ph]].

[55] G. Baym, D. H. Beck, P. Geltenbort and J. Shelton, Phys. Rev. Lett. 121, 061801 (2018) [arXiv:1802.08282 [hep-ph]].

[56] T. F. Motta, P. A. M. Guichon and A. W. Thomas, J. Phys. G 45, no. 5, 05LT01 (2018) [arXiv:1802.08427 [nucl-th]].

[57] J. M. Cline and J. M. Cornell, JHEP 1807, 081 (2018) [arXiv:1803.04961 [hep-ph]].

[58] M. Pfützner and K. Riisager, Phys. Rev. C 97, no. 4, 042501 (2018) [arXiv:1803.01334 [nucl-ex]].

[59] X. Sun et al. [UCNA Collaboration], Phys. Rev. C 97, no. 5, 052501 (2018) [arXiv:1803.10890 [nucl-ex]].

[60] G. K. Karananas and A. Kassiteridis, JCAP 1809, no. 09, 036 (2018) [arXiv:1805.03656 [hep-ph]].

[61] H. Ejiri and J. D. Vergados, J. Phys. G 46, no. 2, 025104 (2019) [arXiv:1805.04477 [hep-ph]].

[62] Z. Berezhiani, arXiv:1807.07906 [hep-ph]. 